\title{
Holistic Integrative Medicine
}

\author{
Daiming Fan and Ningli Wang
}

Many people engaging in medical science asked me, "You've been working on the exploration of Holistic Integrative Medicine (HIM) for quite a while. What is the progress you have made in your research? Have you got your article published? May I be your first reader?" Each time I was confronted with such a situation, I felt embarrassed and speechless. Just as an old saying goes, it is easy to put the boat along with the current but it is difficult to sail against the current [1].

When an author writes an article, he frequently coins a new term or defines a new concept to guide the reader to grasp its key point, just like following the vine to get the melon. This time it is difficult to define holistic integrative medicine in words even though I have a clear idea in my mind [2].

In terms of "integration," we should discuss the universal laws based on which human knowledge arises and develops. Epistemology holds that knowledge and practice are the two essential means for mankind to gain an insight into the world. Practice is not only the basis of knowledge but also the sole criterion to verify the knowledge. Knowledge may be developed from practice and in turn serves practice. During the process of knowing, people discover the nature laws through practice, and again through practice verify and take advantages of the nature laws. Practice, knowledge, again practice, and again knowledge. This form repeats itself in endless

D. Fan $(\bowtie)$

Xijing Hospital of Digestive Diseases, The Fourth Military

Medical University, Xian, China

Chinese Academy of Engineering, Beijing, China

e-mail: fandaim@fmmu.edu.cn

N. Wang

Beijing Institute of Ophthalmology, Beijing Tongren Eye Center, Beijing Tongren Hosptial, Capital Medical University,

Beijing, China

Beijing Ophthalmology and Visual Sciences Key Laboratory,

Beijing, China

e-mail: wningli@vip.163.com cycles, and with each cycle the content of practice and knowledge rises to a higher level. Unity and diversity of the world determine the people's perception of the world. People know the world either from integrated perspective or from differentiated perspective. As a unity of opposites, differentiation and integration oppose each other and complement each other. Such a law is reflected at every stage of the development of science. Differentiation of science refers to the differentiation of one or several comparatively independent disciplines from one essential discipline. Integration of science refers to the overlapping and merging of similar or even different disciplines, which aims at breaking the boundaries of existing disciplines and establishing many marginal or holistic disciplines so that the irrelevant disciplines are closely integrated into an organic body. HIM means that people conduct an organic integration of the most advanced knowledge and theory in medical sciences and the most valuable clinical experiences to establish a new medical system. It will be beneficial to people's health and is effective in medical treatment by implementing unceasing adjustment in accordance with the changes of society, environment, and patient's psychology $[3,4]$. The Chinese character "zheng" (Zen in English) refers to rearrangement which is a strategy focusing on process while the Chinese character "he" refers to fitness which is a standard with the emphasis on result. Therefore, HIM originates from history and philosophy so it should be in conformity with historical trend, scientific laws, and people's will.

The development of the world accords with the phenomenon of "unity after a long time of division and division after a long time of unity," complies with the trend of "spiral development," and abides by the law of "the negation of negation" and "the unity of opposites," which embodies the philosophical concept that "one divides into two" and "two combine into one." With too long or too tight unity, new things will be difficult to emerge and social development will be blocked. With too long or too frequent division, there will be no cohesive force and the motivation of making progress. It is true to nature although no noticeable changes take place in the world 
in a certain period of time (maybe thousands of years). Either division or unity is just what people view things from different perspectives. The essence of the world lies in the law of unity and division. For example, it is self-evident that there are cells and organs in human body and they are indispensable. However, with the explosion of human knowledge, "organ-based theory," "cell-based theory," and even "genebased theory" were proposed. Just as the rivers join and split, whether we make a division or achieve a unity should comply with the laws of nature, which is "the theory of division and unity" proposed in this chapter.

The development of medicine is a kernel part of scientific advancement in the world and abides by the laws of nature. In the early stage of medical development during the early period of human development, people who were short of practical experience and knowledge explored the unknown areas, such as the mysterious phenomena related to their life and health through primitive social activities and backward productive forces. The dispersed, sporadic, and personal experience was collected and compiled into several books which were passed down from the masters to their apprentices, particularly from father to his sons. Unity is a typical feature of this period, emphasizing the concept that "two combine into one." For example, the rich knowledge of traditional Chinese medicine was compiled into three books:

Huangdi Neijing, which is related to preclinical medicine; Shanghan Zabing Lun, which is concerned with clinical medicine; and Shennong's Herbal, which is associated with pharmacology. Medical development in this period is mainly characterized by integration. The early practice of medical pioneers constructed the tentative framework of traditional Chinese medicine permeated with the concept of "the unity of man and nature," namely, man is an integrated part of nature and vice versa. "Those who submit will prosper, those who resist will perish." Several well-known medical masters such as Bian Que, Hua Tuo, and Zhang Zhongjing accumulated a large amount of medical knowledge through practice. Bian Que, who is said to have lived more than 300 years, wrote two books entitled Bian Que Neijing and Bian Que Waijing. In fact, he did not live to that old age. It is people who attributed 300 years' contributions of medical workers to him, which embodies the concept that "two combine into one" during the early medical development and is somewhat similar to holistic integrative medicine in this chapter or primary integrated medicine.

As time went by, with the accumulation of experience and knowledge and with the increase of more famous doctors who had unique skills, a relatively systematic framework of traditional Chinese medicine was established, which is a valuable knowledge reservoir of Chinese medicine and pharmacology [5]. However, integrated medicine gradually developed into special medical disciplines since Qin Dynasty and was divided into internal medicine, surgery, gynecology, pediatrics, and other disciplines in Han Dynasty and Tang
Dynasty. Meanwhile, pharmacology developed with the discovery of more traditional Chinese herbs and their properties and effects. The formulae in the book Thousand Pieces of Gold Formulae were said to have the effect of "treating any diseases." Newly Revised Materia Medica, the first pharmacopoeia in the world, was published in Tang Dynasty.

Both TCM and Western medicine serve the same purpose in two separate ways. Although Western medicine has also developed following the trend of division, its developmental speed and degree are more apparent than TCM. Because Western philosophy and Chinese philosophy have different origins, Western medicine puts more emphasis on evidencebased practice, qualitative study, and quantitative study at the cellular and molecular levels, while TCM takes all factors into consideration and makes a systematic study at the macro-level. Both of them have made great contributions to world medicine [6-8]. (Detailed discussion of this aspect will be omitted for the limited length of this chapter.) However, it should be particularly pointed out that medicine has made a rapid progress from macro-level to microlevel since Antonie van Leeuwenhoek invented a microscope in the seventeenth century and then medicine was divided into preclinical medicine, clinical medicine, and preventive medicine. In preclinical medicine, the structure of human body is divided into different systems, each of which consists of some organs. Each organ is further divided into different tissues composed of cells, organelles, molecules (protein, DNA, and RNA), etc. As for clinical medicine, it is divided into internal medicine, surgery, and medical specialized disciplines and then it is divided into gastroenterology, hematology, cardiology, orthopedics, general surgery, urology, etc., which are called third-tier/level disciplines.

Many third-tier/level disciplines are divided into more specialized ones in recent 10-20 years. I wonder whether they could be called fourth-tier/level disciplines. For instance, orthopedics department consists of several divisions concerning spine, joints, limbs, etc. Gastroenterology department is composed of different divisions for the treatment of gastrointestinal, liver, colorectal, pancreatic diseases, etc. In addition, the former Chinese Society of Gastroenterology has been divided into current Chinese Society of Gastroenterology, Chinese Society of Hepatology, and Chinese Society of Digestive Endoscopy. Accordingly, the former Chinese Journal of Digestion has been divided into today's Chinese Journal of Digestion, Chinese Journal of Hepatology, Chinese Journal of Digestive Endoscopy, and Chinese Journal of Pancreatology. At present, a so-called fourth-tier/level discipline has been divided into many cooperative groups, with the number amounting to more than ten. Furthermore, some people propose that surgery should be further divided into more specialized divisions. I wonder how specialized the division should be. It seems that one will never give up if the whole human body is not broken into pieces, with his head decapi- 
tated, his heart and lungs torn up, his spleen and stomach separated, and his liver and intestines cut into inches, which is one of the features of modern medicine.

It is true that the unceasing division has enhanced the development of modern medicine. Nowadays people have had a better understanding of their bodies and have acquired much more medical knowledge, and the medical treatment has become more effective. There is no denying that the therapeutic effect has been enhanced and people's life span has been increased. However, we also admit that division of medicine has brought some disadvantages, detriment, and even disastrous effects, which are reflected in the following nine aspects.

\subsection{Patients Are Treated as Organs}

With the more specialized division of clinical departments, doctors have a dim impression of general medicine. Compared with the experienced doctors, the green hands have no access to the training of integrated medicine. As a result, they only have a command of medical knowledge concerning the fourth-tier/level disciplines and a part of human body. Consciously or unconsciously, they see their patient as an organ. For example, they treat a patient with liver cancer as a cancerous liver, namely, they pay more attention to carcinoma rather than the patient. When cancer cells were found in the abdominal cavity of a patient, some doctors tried to discover the primary carcinoma. Sometimes they ended in vain even after the autopsy of the dead patient. In fact, "a cancer patient" should be regarded as "a patient who has contracted cancer" rather than "cancer contracted by a patient." When they provide him or her with treatment and care, doctors and nurses should put emphasis on the patient himself or herself. They should take their patient as a person with physical, psychological, and social needs rather than focusing on his or her organ with physiological or pathological changes. Different people with the same cancer may turn out to have different fates. Some patients with cancer died even though they had their tumor removed, or had their cancerous organs fully cut off, or even had all the surrounding lymph glands removed no matter whether there was a metastasis or not. On the contrary, some patients with cancer survived even though their cancer had not been radically cured or even had not been treated. Here are some other examples. Some patients who were not informed of their advanced cancer still work as usual and even take part in a sports meet as a runner. In contrast, some patients who were informed of their cancer at early stage did not eat and drink for several days in a state of anxiety and were scared to death only in a few days. These examples demonstrate that many doctors only took care of the organs relevant to their specialties and the diseased organs or lesion, so they got the dis- eased organs cured but damaged other organs crucial for a patient's survival. They couldn't attend to one thing without neglecting the other. Some doctors who did not realize that topical lesion is the signal of a systemic disease or indicates the patient's poor health only focused their treatment on topical lesion. Consequently, the disease did not get cured and the patient died.

\subsection{Symptoms Play a Dominant Role in Diagnosing Disease}

Symptoms are the main indicators to reflect the expression, severity, progress, and outcome of a disease. However, the same symptom may be shown in different diseases while different symptoms in the same disease. Moreover, the sequence of the symptom manifestation of the same disease in different patients may vary. Some diseases, though very serious, have no corresponding symptoms at all until the advanced stage. Nevertheless, some doctors use a stereotyped approach to the understanding of the disease, comparing its symptoms with what is stated in their textbooks, so they are figuratively called "Dr. Symptom," which means they make a diagnose and treatment of the disease only based on their observation of its symptoms. For example, the doctor gave a patient with severe abdominal pain an injection of highly powerful narcotics, which resulted in the relief of the pain but the patient died in the end. In fact, the pain is just the early symptom of the disease but more severe changes are taking place or will take place in his or her body. The administration of painkillers at will may mask the real cause of the disease and even delay the treatment for the patient. Symptoms, which are various and are always changing, comprise the superficial ones and substantive ones. For an experienced doctor who treated a patient with eight symptoms, he would focus his or her attention on one substantive symptom, and the other symptoms would soon disappear as the result of the proper treatment to the substantive one. In this way, this skillful doctor saved the patient's life. When an inexperienced doctor treated a patient, he or she would administer treatment for each symptom. After the administration of a great amount of drugs, most or even all of the symptoms like cough, fever, and abdominal pain disappeared. As a result, the patient who looked pale and remained in a deep coma died of liver failure due to the overuse of drugs.

\subsection{Clinical Examination Plays a Key Role in Diagnosing Disease}

Major clinical examinations, such as laboratory testing, medical imaging, and pathological diagnosis, play an important role in the development of clinical medicine and the 
practice of conventional treatment. It is true that a hospital cannot be modernized without the rapid development of clinical examination. Likewise, a modern hospital must be equipped with advanced Laboratory Testing Department and Medical Imaging Department. Just as a cleverest housewife can't cook a meal without rice, a clinician with exquisite skills cannot perform his or her tasks in a hospital without them. However, many clinicians pay no attention to the training and practice of the basic diagnostic skills such as "inspection, touching, knocking and acouophonia," or "observation, listening, interrogation and pulse-taking." When a patient with fractured hand and leg went to the hospital, the doctor asked him or her to have his or her bones X-rayed in medical imaging department. After examining the results of CT and MRI, the doctor informed the patient of his or her fractured hand and leg which the patient himself or herself had already known. What's worse, some doctors diagnosed and even treated the disease based on the results of X-ray image, ultrasound scan, blood tests, etc., turning blind to the patient. I once examined the CT image of a patient who was treated in another hospital. I diagnosed the patient as having cirrhosis of the liver by examining his CT image from another hospital. However, the doctor in that hospital diagnosed it as liver cancer and administered percutaneous transhepatic variceal embolization (PTVE) and local chemotherapy to him. Isn't it another disaster fallen upon him when PTVE and local chemotherapy were administered to him who had less normal tissue due to the cirrhosis of the liver? The hospital defended itself in this way: since the patient had already had hepatitis virus infection, PTVE could kill the virus though it had little effect on cancer cells. What a ridiculous explanation! Some young doctors are so dependent on clinical examination in their diagnosis of the disease that they can't carry out their practice without them. They would track down any possible hints of disease through countless examinations so as not to miss them even if the examination results were proven futile and ineffective. If the values of the tests were found on the increase or abnormal image or cells were detected in the patient, he or she was diagnosed as having some disease, and vice versa. Therefore, clinicians have become a slave to clinical examination because they give it first priority in the whole process of practice, including diagnosis of the disease and administration of medicine. In fact, the condition of human body is changing all the time. There are many cases like "the same disease showing different images," "the different diseases showing the same image," "one disease showing many images," and "many diseases showing no image." Once I acted as a supervisor in a reexamination of the postgraduate candidates. I asked them a question "Under what condition will carcinoembryonic antigen (CEA) increase?" They gave three different answers: it would increase if the patient had a cancer, or if she was pregnant, or if he smoked. "Any other possible cause for the increase?" I asked. "None.
They are all the answers in our text books." At this moment, one student said that CEA would also increase if something was wrong with examination. It is a quite good answer, totally different from the answer to the question "Under what condition does 1 plus 1 equal 3?" put forward in the intelligence game by a famous Chinese comedian Benshan Zhao. It is this philosophical way of thinking that paved the way for the young man's later success. All the doctors should cultivate this way of thinking. In fact, suffering from cancer, being pregnant, or smoking does not necessarily contribute to the increase of CEA while the decrease of CEA may be related to one of the three cases, showing the complex mechanism of human body. Cancer indicators are not necessarily the determinants of cancer. No absolute thing exists in the world, nor does absolute value exist in medicine. If it does happen, it is due to the small size of the collected samples. During the period of SARS outbreak, a protein detected in SARS victims was found to be $100 \%$ positive, but $100 \%$ negative in normal people. The finding was taken as a novel approach to detecting SARS virus. Later it turned out that the protein in SARS patient was only related to fever, which can be caused by different diseases with protein positive. Therefore, this protein cannot be regarded as specific to SARS diagnosis. Isn't it so complicated if that protein is only used for diagnosing fever? Clinical thermometer or even our hands and eyes do work.

\subsection{Doctors Act as Pharmacists}

Drug treatment has been considered as an important means of curing diseases and an indispensable part of medical practice since ancient times. Medical treatment and drugs are so closely related to each other that they are figuratively called "one family." However, there is a great variety of drugs in the market. For instance, cephalosporin, one type of antibiotics, is used in some hospitals with more than 20 types. The same drug not only has different chemical names, brands, and dosage but also shows different efficacy and side effects after administration, which makes the doctors puzzled at their medication. In addition, most doctors would like to give prescription based on the symptoms rather than the disease itself. The first symptom or the most serious one will be treated as priority. One drug will be used to treat one symptom and if it doesn't work another drug or even several drugs will be considered as alternatives with the help of pharmacists. In some cases the symptom worsens; in other cases the symptom disappears but another symptom occurs. What's worse, all his or her symptoms disappear but the patient dies of adverse reaction to drugs. When confronted with such complex cases, the doctors would count on the drug rather than their diagnosis and treatment. Consequently, it is the pharmacist who instructs the doctor to prescribe drug, caus- 
ing great confusion in treatment. Once a doctor prescribed erythromycin for a patient with fever, and when the drug was not available in dispensary, the pharmacist dispensed daunorubicin instead. When the nurse asked whether the drug was wrongly dispensed, the pharmacist explained that it was erythromycin of a new generation. In fact, daunorubicin is a kind of anticancer drug. In Chinese, erythromycin is called "Hong Mei Su" and daunorubicin "Rou Hong Mei Su." As a result, a single word variation in the drug name makes the great difference. Here is another story. A patient suffered from liver dysfunction after stents were embedded in his heart. He was taking some drugs when I visited him to hold the consultation. I was surprised to find that he was taking 16 types of tablets, coupled with the damp-removing painkilling ointment. Why did he take so many drugs? The real reason was that because he was a leader of the university, directors of each department showed their great concern for him by prescribing the so-called goodwill drugs, namely, the drugs for prevention. As a result, the drugs heaped up. It is not wrong to prescribe the drugs from their own individual professional angle, but every drug is more or less toxic to some degree and the accumulated toxicity caused great damage to the patient's liver, which almost led him to death. I asked him to stop taking all the drugs except for aspirin for anticoagulation, and finally the patient recovered. The cases indicate that drug administration should be scientific. Traditional Chinese medicine stresses treating diseases in a dialectical way. Chinese medicines are grouped into "Monarch, Minster, Assistant, and Guide." Reasonable matching is the key point. Traditional Chinese medicine stresses the different roles played by different ingredients in a prescription. If the matching is not "One, Three and Five," there must be "One, Five and Seven." One refers to the main ingredient, or called monarch ingredient, to treat the major disease; the minister, assistant, and guide ingredients must be added or subtracted in terms of the symptoms to treat the disease in cooperation with the main ingredient. If a medicine has a conflict with the main ingredients, it may not cure the patient's disease, but worsen his or her disease. Probably, the disease is not cured, but the patient may not lose his or her life.

\subsection{Physiological Treatment Is Separated from Psychological Treatment}

A patient should be regarded as "a person with a disease or diseases." So not only the "disease" should be cured but also the "person" should be cared about. Although the pathological change in his or her body can be perceived, the psychological suffering is neglected. His or her recovery involves not only the doctor's treatment but also the patient's efforts, without which the disease may relapse or his or her condition may worsen [9]. For example, a young lovelorn lady jumped off a building and was severely injured. The injury might heal through medical treatment but if the psychological problem was not tackled thoroughly, she was likely to commit suicide again by hanging or drowning herself. So both physical and psychological treatment should be applied for her full recovery. With more and more cases like that, it would be impossible to cure all these patients even though we have more doctors.

In the early stage of medical development, owing to low productivity and lack of scientific knowledge, one could not understand the real cause of the disease. At that time psychological consultation played a significant role in the treatment of diseases. The person engaged in this practice was called "witch" or "witch-doctor." There was once a "witchdominated" era when the role of psychological treatment was so overstressed that people were obsessed with gods and ghosts, resulting in the prevalence of superstitions. The overemphasis on the psychological treatment is totally wrong. To the other extreme, with the development of medical science, psychological treatment was disregarded and even completely denied at a time. Take Chinese Cultural Revolution for example. The campaigns of "Doing away with four olds (old idea, old culture, old custom and old habit), Rooting out the feudal culture, Sweeping away the ghosts and monsters, and Opposing metaphysics" were waged to take the nonmaterial things as pseudoscience, false medicine or idealism, or even ideological problem. Up to now, such ideas, views, and practices are still occurring everywhere and even at the present time. In fact, even if symptoms and even serious ones are shown in some diseases, they cannot be detected through the medical examination, because they are caused by psychological disorders rather than the pathological changes. Curing the mental worries can relieve the symptoms, or even cure the disease. About 30-40\% of outpatients with digestive disease recovered in this way. Besides, some psychological disorders caused by organic disease do more harm to the patients than the disease itself. Psychological disorder will be cured as the result of effective treatment of organic disease. In short, psychological disorder or physiological disease sometimes exists independently, but most of the time they coexist and interact as cause and effect. From the perspective of holistic integrative medicine, only when doctors are armed with both medical and psychological knowledge can they treat the intractable diseases.

\subsection{Medical Treatment Is Not Well Coordinated with Nursing}

The Guideline of National Medical and Health System Reform points out that nursing reform aims at changing the nursing mode, namely, switching "the disease-oriented functional nursing" to "patient-oriented primary nursing" to offer 
patients continuous and seamless high-quality nursing services. The old saying "Treatment and nursing account for 30 and $70 \%$ in the patients' recovery respectively" highlights the importance of nursing in the disease treatment and patients' recovery. The patients hope to get not only the effective treatment but the holistic nursing as well. Generally speaking, the process of diagnosis and treatment for patients might be short and unconscious while their experience of nursing services is long and conscious. The medical service quality has been greatly enhanced with the involvement of nursing and its staff in the medical service. This transformation and the development of holistic nursing have changed the primary-secondary relationship between doctors and nurses into an equal and cooperative one for patients' recovery [10]. The successful operation lies in the crossdisciplinary cooperation and coordination among surgeons, anesthetists, and nurses. On some occasions, nursing even plays a more important role. Nurses, stereotyped as working under the guidance of doctors, are now supposed to make arrangements for surgeons and anesthetists in the operation room, and supervise them to operate in a standardized way to avoid complications, even medical accidents. In foreign countries, nursing and nursing staff are greatly valued by the doctors and administrative staff and highly respected by patients. However, in China, this new type of cooperative relationship between doctors and nurses has not yet been fully recognized. In fact, nurses should perform the frontline tasks such as drug administration or their observation of patients. However, because of their different traditional roles, doctors and nurses are not well coordinated in such aspects as knowledge mergence in medical teaching and learning, technical coordination, professional complementation, and intercommunication. For example, nursing is not included as a whole in the clinical treatment. Nurses are not asked to participate in case discussion, preoperative discussion, or death case discussion, and even if asked occasionally they only act as foils. As a result, nurses fail to know their essential duty in rescuing seriously ill patients. What's worse, lack of cooperation between doctors and nurses may even cause medical disputes or medical negligence. As mentioned above, "doctors act as pharmacists," and "pharmacists act as drug dispensers." What about the nurses? Nurses act as "nurses who deliver drugs." Most of their working hours are spent on non-nursing work, such as drug dispensation and delivery instead of nursing by the bed. They carry out a variety of medication orders mechanically without any attempt to communicate with the doctors about the patients' condition or treatment. They account for the hospitalization expenses and press for payment rather than offering patients the recovery and psychological consultation. Consequently, doctors and nurses have fewer ward inspections together, and doctors seldom inform nurses of the remedies. So, the cooperation between doctor and nurse is compromised and even clashed. Nurses keep occupied by such physical labor as delivering drugs, giving injections, taking temperatures, and counting pulses, a far cry from the requirements of holistic nursing. Doctors always assign the nursing degree to nurses in their medical orders simply based on the severity of the disease, without considering patients' nursing needs, or other psychological and social factors. For example, when a patient with tertiary care who has almost recovered from a major surgery may suddenly die of pulmonary embolism, it is nurses, rather than doctors, that are usually blamed for their improper inspection. So the nurses have become "the accused." It is obvious that direct cause of this case is the incompatibility of nursing degree with the patient's condition. Since the ancient times, there has been the concept of "integrated medical care." Hippocrates, known as "the father of medicine" by ancient Greeks, used to teach the patients how to gargle and teach the nephropathy patients how to have a rational diet, which developed into "oral care" and "diet nursing" in modern nursing. Shizhen Li, the author of "An Outline Treatise of Medical Herbs," was good at both treating and nursing. He decocted medicinal herbs for the patients and fed them in person, which has become a muchtold tale. This is called the "oral administration" in modern nursing. I do not mean that doctors should do nurses' job, or vice versa. Both of them should fulfill their own duty. The point is that doctors should carry out their medical service from the perspective of nursing while nurses from the perspective of treatment.

\subsection{Western Medicine Conflicts with Traditional Chinese Medicine}

Western medicine and traditional Chinese medicine have developed for a thousand or even thousands of years, even though they belong to different medical schools. As the common wealth of human civilization, both of them have contributed to the survival, reproduction, and development of mankind, though there are some similarities and dissimilarities. However, with their respective features in theories and practices, both of them tend to emphasize their own specialties and advantages. If they are reconciled for the same goal of diagnosing and treating diseases, they could be enriched mutually to form an integrated Western-Chinese medical system, getting the twice results with the half efforts $[11,12]$. Unfortunately, their coordination has never made any significant improvement due to constant mutual condemnation and contradiction. In history, Western medicine practitioners looked down upon traditional Chinese medicine practitioners. Here are some examples. Around 1880, the new practitioners of TCM were against the senior ones; with the introduction of Western medicine into China during the Period of Northern Warlords Government, the practitio- 
ners of TCM were excluded by the doctors; and until now, they are still negated in society. Some people even openly claim that "traditional Chinese medicine is not scientific." In fact, it is one-sided to put emphasis on the correctness and contribution of either traditional Chinese medicine or Western medicine. For example, Western medicine has advantages in curing acute illnesses while Chinese medicine in curing chronic ones. Western medicine focuses on nidus for the immediate relief with medication or operation, while Chinese medicine treats the body as a whole in order to achieve an overall recuperation, regain vigor, and finally reach the state that "Vital energy exists inside, so pathogenic factors can not prevail." For instance, at present the best medicine for ulcer is proton pump inhibitors with an immediate effect and a rather high cure rate. However, for functional dyspepsia, Lenitive Pill (Baohe Wan) or Agastachis Pill for restoring healthy energy (Huoxiang Zhengqi Wan) may be more effective, since proton pump inhibitors cannot maintain a long-term effect on it. If we give the patients ulcer proton pump inhibitors at the acute phase and Agastachis Pill for restoring healthy energy at the recovery phase, the effect of the treatment will be much better with the integrated methods.

\subsection{Treatment Is Prior to Prevention}

It is well known but far from well accepted that public health service should mainly focus on the prevention of diseases. It is said that Que Bian, the ancient Chinese medical master, had two elder brothers. Que Bian himself specialized in treating advanced diseases, which earned him great and eternal fame. His second elder brother specialized in treating early diseases, and his eldest brother specialized in treating potential diseases, which is similar to modern preventive medicine. Because of the differences in their specialties, Que Bian's two elder brothers had never gained their fame and still remain unknown. We have no evidence to confirm this story, but one thing is certain that from ancient times to the present, preventive medicine has never aroused enough attention. Even if it did arouse "enough" attention, it is not comparable to that by preclinical medicine, not to mention clinical medicine. In fact, a disease is like bursting flood when it occurs. Which is more important, going downstream for flood fighting and rescuing people or plugging the breach on river banks immediately? The answer is definite. Preventive medicine should have been integrated medicine. The specialization of modern medicine turns the linear thinking pattern of "one disease, one gene; one pathogen, one treatment" into the mainstream in medical development. Public health incidents, such as SARS and H1N1 influenza, have proved that this linear longitudinal treatment is not enough. Instead, we need a comprehensive "point-line surface-body" prevention and treatment, taking "pathogen, disease, population and society" into consideration. Clinical medicine mainly involves the diagnosis and treatment of a disease while the preclinical medicine studies the nature of a disease, but neither of them can prevent such a disease from recurring among different populations in different regions. Based upon the study on factors influencing the health and their effects, preventive medicine aims at illustrating the interrelation between external environment and public health, and subsequently laying down some strategies and measures for prevention [13]. Therefore, preventive medicine itself needs to be integrated with not only the disciplines in preventive medicine, such as epidemiology and labor and environmental health, but also the disciplines in preclinical medicine, clinical medicine, and even social medicine.

It was once believed that with the accomplishment of Human Genome Project, people could discover the secrets of life and disease, and conquer all the diseases [14, 15]. However, the reality is not in line with our expectation. The reason is that genes can only predetermine the genetic predisposition to certain diseases, while most diseases result from the combined effects of environmental factors and organic factors. It is true that preclinical medicine uncovers the pathogenesis of diseases while clinical medicine offers treatment to diseases. But we have to turn to preventive medicine for effective prevention from the "postnatal noxious stimulation" to nip the problem in the bud. When it comes to preventive medicine, people can't help thinking of an emergency with staffs in their protective gear, shouldering a sterilizer and spraying disinfectant everywhere. In fact, this is totally misunderstood. Preventive medicine does much more than that. It can not only offer treatment during and after the incident, but also, more importantly, provide prevention in advance. In short, it can curb not only advanced diseases, but also potential diseases in advance. Suppose a football team of "medicine" in a football match. During the match, clinical medicine acts as the forwards, preclinical medicine acts as coaches to make tactical plans, and preventive medicine serves as the goalkeeper. If the team has a top goalkeeper, the external factors, such as playing home or away, or "black whistles," could do little to the result. So such a team will be unbeatable. Accordingly, preventive medicine should also play a pre- and post-role in integrated medicine. That is, preliminary research must be conducted as the highest priority, and later intervention must be carried on in time and persistently. Therefore, preventive medicine should always run through integrated medicine.

However, the current preventive medicine is clearly separated from clinical medicine and preclinical medicine, and the situation is worsening. Only with the comprehensive integration of preclinical medicine and clinical medicine can preventive medicine offer effective strategies to disease prevention. However, at present the science of medicine is 
becoming more and more specialized, which really hinders the development of preventive medicine in every aspect. If this situation continues with treatment over prevention, it is likely that the task, originally accomplished by one doctor in preventive medicine, will require 100 clinical doctors at present. If this tendency goes on, ultimately 1000 or even 10,000 clinical doctors may not be able to solve the problem which one staff in preventive medicine can handle at the early stage.

\subsection{Gap Between Urban and Rural Medical Service Is Widening}

In China, there exists a huge urban-rural gap in medical service, causing a serious social problem [16]. As a result of the unreasonable distribution of health resources under the current Chinese health system rather than the overspecialization of medicine, this problem is different from the previous eight ones. Only with the integration of medicine can this problem be solved. At present, most of the doctors in rural areas, with the title of general practitioners, are not qualified and doctors in urban areas are mostly specialists without enough competence for general medical service. This situation has resulted from the urban-rural economic gap, the unbalanced hospital distribution, and especially the specialization of urban hospitals over the past several years. On the one hand, rural practitioners desire to work in urban hospitals but they are not competent at special medical work. On the other hand, specialists in urban hospitals are not only unqualified for the general medical work in rural hospitals but also unwilling to work there because of the poor economic and living environment. Consequently, a large number of rural patients are pouring into cities for better medical service, imposing a heavy burden on national transportation. In rural areas quality medical service is not available, directly contributing to inadequate and overly expensive medical services in China. In addition, technical secondary schools and 3-year colleges were banned, which used to train general medical practitioners for rural hospitals. Even though both the central and the local governments are promoting healthcare reform aiming at solving this problem, there is still a long way to go. To sum up, the increasing labor division in society has largely improved the proficiency of people's professional techniques, working efficiency and social prosperity, and living standards. Likewise, the increasing specialization of medicine has greatly promoted the development of medical techniques and the doctors' professional ability. However, with the change of lifestyle and disease spectrum, the specialization has seemingly reached its limit, suggesting that endless "specialization" cannot solve the current medical problems. Unless the problems get settled, the development of modern medicine would not only be hindered, but slip off the track as well. Then, what should we do? We should promote theoretical study on integrated medicine and accelerate the practice of holistic integrative medicine [17].

\subsection{Promoting Theoretical Study on Integrated Medicine}

To promote researches in integrated medical theories, we must first clarify the following points and set the priorities in theoretical research.

1. With the advance in medical research, the causes of some diseases have been identified. However, in clinic, most of the diseases cannot be attributed to a specific cause. For example, we cannot identify the specific cause of such diseases as primary hypertension and autoimmune because they result from combined effects of several factors. A trauma may even induce the change of more than one system or an organ. Therefore, it is unlikely to know and cure a disease only by one discipline.

2. With the shift of lifestyle, some diseases like cancer and diabetes become incurable to some degree. Specialized treatments may lead to poor life quality and relatively short-term survival, which to some extent may compromise human dignity. Nonetheless, with integrated medical treatments, we can help these patients live with disease and improve their life quality. Integrated medicine not only shows a respect to human dignity, but is a must for humans as well.

3. With the change of natural environment, emerging and re-emerging infectious diseases, like AIDS, SARS, and A (H1N1) influenza, are becoming a threat to humans due to the delay of induced resistance to the diseases. Doctors have such little knowledge about the cause and mechanism of diseases that they could do nothing, much less diagnose and treat the diseases. One single medical discipline is only a drop in the bucket, which cannot win the war against the diseases. To win the war requires the multidisciplinary integration.

4. With the advent of the ageing stage, the average life span of Chinese people has increased by more than 30 years during the past 50 years. During these additional 30 years, the physical condition of Chinese people will change as a result of the interplay with the nature and physical senescence. These physiological or pathological changes still remain uncertain or under-investigated. It needs multidisciplinary integration to resolve the present and future medical problems caused by ageing.

5. With the development of medical technology, many diagnostic techniques and therapies, which were beyond imagination in the past, have already been applied to treat many thorny cases. However, the development of medi- 
cine seems to have hit its bottleneck. The classical medical techniques have been proved effective in solving only local problems rather than global or systemic ones. The sustainable development of medicine must count on the integration of medical knowledge and technology.

6. With the march of modern society, the incidence of disease is increasingly related to social reality which triggers a variety of psychological problems. If doctors only focus on physical diseases and ignore psychological problems, it is by no means to treat or cure a large number of patients even though we have more hospitals and doctors.

Integrated medicine is a reformation of the traditional medicine, symbolizing a new stage of development from specialization to integration in the medical course. It is not a regression but a progress. It aims at achieving the following goals: the integration of biological factors and that of psychological, social, and environmental factors, and the integration of the most advanced medical discoveries in all life-related areas and that of the most effective clinical experience in all medical specialties. It also requires us to analyze a problem with not only linear, one-dimensional way of thinking in natural science, but also nonlinear multidimensional way of thinking in philosophy. Through this thinking mode and the reintegration of the above four integrations, a more comprehensive, more systemic, and more scientific new medical knowledge system would be established, which accords with natural laws, health maintenance, and disease prevention, diagnosis, and treatment. This is the unity of "rearrangement" and "combination." In this sense, integrated medicine should be defined as holistic integrative medicine (HIM). Although HIM has not yet been fully recognized, it will become a worldwide trend and international frontier of medical investigation, which will be definitely tough and complicated. As we all know, it has already taken thousands of years for both traditional Chinese medicine and Western medicine to evolve from generalization to specialization, with the former lagging behind the latter on the way. Therefore, integration is doomed to be difficult because it defies traditional theories, established practices, academic authority, and force of habit. In addition, medicine has developed into an enormous theoretical system and will keep growing. In this system, we have to differentiate the primary from the secondary, the cause from the effect, the predecessor from the successor, and the truth from the false. We should try to attain the essence out of the dross and strip the false off the true by analyzing the nature of the problem and its relevant factors. In the era of knowledge explosion, what to be integrated and how to integrate are worthy of extensive and intensive study. The more we want to integrate, the more complex the integration is. Therefore, we should take scientific methods and forward-looking strategies to achieve the integration in the historical context. The outcomes of inte- gration must be able to stand the test of practice. We need to highlight the advantages of promoting HIM and point out the disadvantages of impeding it, and establish excellent models of promoting HIM so that we can wipe out the stereotypes completely and scientifically and form new concepts. In the promotion of HIM, we should theoretically illuminate that everything, regardless of whether it is as large as the universe or as small as human body, should be regarded as a system within which all internal elements are closely related. This idea requires doctors to have a global picture instead of only a local one.

Although there are some similarities between HIM and general practice, their differences are marked. General practice requires general practitioners to be expert in one field and versatile in others. But their competence developed from the established basic theories and common practice is only the sum of ordinary abilities, like the sum of A + B + C. HIM emphasizes rational and scientific integration of the most advanced theories and the most effective experience, which is like the multiplication of $\mathrm{A} \times \mathrm{B} \times \mathrm{C}$. The former is a quantitative increase which can be achieved by ordinary people while the latter indicates a qualitative leap which can be achieved by only a few talents. For instance, Specialist A knows how to rub red lotion with high concentration. Specialist B knows how to rub blue lotion with high concentration. General Practitioner $\mathrm{C}$ can rub both the red and blue lotions but with lower concentration. But Doctor D was so well trained with HIM that he or she can invent green lotion by integrating red with blue lotion with the effect of both lotions, which can be interpreted as "Green comes from red and blue but is better than both."

Although there are some similarities between HIM and translational medicine (TM), their differences are evident. TM translates preclinical findings into clinical application to test their values and then optimizes the preclinical research [18]. It finally takes the advantage of preclinical findings to improve human health through the process.

HIM is different from complementary and alternative medicine (CAM). CAM views Western medicine as mainstream medicine and underestimates other schools of medicine as nonmainstream medicine. CAM is a school discriminating against the viewpoints of other schools [19].

Although there are some similarities between HIM and evidence-based medicine (EBM), their differences are obvious. EBM lays stress on making rational clinical decisions which may result in desirable efficacy and fewer side effects on the basis of the available evidence from the investigation of a group of patients [20], while HIM represents the cognition of human health and diseases with emphasis on comparison, analysis, and integration of theories and practice. HIM aims at exploring the most optimal therapeutic methods with the best curative effects and establishing a new medical knowledge system. 
The transformation of the medical model is an extremely complicated project. We need to define HIM with high accuracy by carefully clarifying its connotation and denotation. In order to define HIM accurately, we should revise, modify, and improve connotation and denotation through discussion. On the one hand, medical workers should be encouraged to realize the urgency and importance of promoting HIM. It is known that the changes of more than one type of genes and cells or one organ take place in the development of a certain disease. The strong regulatory and protective mechanism may cause overall changes of the functions and structures of the systems in human body. In addition, the development and outcome of the changes are related to environment, dietary habit, and even interpersonal relationship. So it is necessary to diagnose and treat diseases with comprehensive consideration of HIM. On the other hand, it is noteworthy that we should not deny the importance of medical specialization [21]. Specialists are and will be playing their part for their therapeutic efficacy and accuracy at present and in quite a long period afterwards.

\subsection{Accelerating the Advancement of HIM Practice}

Truth can only be verified through practice. HIM is both a profound and practical science, which needs to be unceasingly enriched, improved, and verified in practice. It is a never-ending process from theory to practice, back to theory, and again to practice. We should adopt the following strategies to accelerate this process $[1,17]$.

\subsubsection{Organizing Academic Conferences on HIM}

The conferences aim at popularizing the concept of HIM, exchanging academic achievements on HIM, and drawing on the experience of HIM practice. At the initial stage, we could hold seminars based on certain topics or certain diseases, such as holistic prevention and control areas for hepatitis B, or holistic prevention strategies for tumor. We could invite the scholars in translational medicine, clinical medicine, and preventive medicine to the seminars and foster discussions on the theory, the diagnostic and therapeutic methods, and the preventive strategies from different perspectives, so that we could reach an agreement and make some guidelines, which will be amended or improved step by step. Based on the experience gained from the seminars, we could hold national HIM conferences. In this way, we could solve the problem that scholars from different disciplines never contact with each other, which is similar to the case that people who are nourished by the same river never greet each other.

\subsubsection{Establishing HIM Academic Associations}

We should attract the talents in HIM and invite the specialists in preclinical medicine, clinical medicine, and preventive medicine to join the HIM academic associations to promote the development of HIM. We could set up the Holistic Integrative Medicine Association of Chinese Medical Association, officially named the Chinese Association of Holistic Integrative Medicine, which could have several branches such as Integrated Gastroenterology Society and Integrated Cardiology Society. In this way we could treat a disease with different methods from different specialties to avoid the phenomenon that "scholars tend to scorn each other."

\subsubsection{Publishing the Journals on HIM}

We should start the publication of Journal of Chinese Holistic Integrative Medicine and its affiliated journals such as Integrated Gastroenterology Journal, Integrated Cardiology Journal, and so on to report the latest achievements on HIM in every discipline. In this way we could gradually deal with the problem that when a dispute over an academic issue arises, only a person of authority has the final say.

\subsubsection{Publishing a Series of Books, Textbooks, or Monographs on HIM}

The publication of these books could solve the problem that many books are various in cover but similar in content due to the fact that the authors copy one another.

\subsubsection{Setting Up HIM Institutes}

We should carry out in-depth specialized research in HIM using techniques for network information analysis as the main approach in addition to the methods applied in the evidence-based medicine. As mentioned above, HIM aims at integrating the most advanced theoretical achievements in every field with the most effective clinical experience in each discipline in a systematic way and achieving the effect of "multiplication" rather than the effect of "addition." However, over the past 200 years, both world population and medical knowledge have been growing exponentially. For example, in the 1980s, the number of biomedical journals worldwide reached 40,000 and is predicted to double every 20 years. Here is another example. Knowledge is outdated at a faster pace than before. In the eighteenth century a person's knowledge became outdated and obsolete in 100 years but now it is in 5 years that a person's knowledge should be 
updated. In the coming 20 years, the amount of knowledge in biomedicine alone will be equivalent to a total amount of knowledge in all fields over the past 2000 years. It is selfevident that human brain can neither "memorize" all the information nor "keep up with" the growth of knowledge. Although the computer cannot rival the human brain in terms of intelligence, it can easily beat the human brain in memory capacity and logic operations. With information integration, we could optimize the integration of the most advanced medical knowledge from various disciplines, and the most effective clinical experience in every specialty, thereby boosting the reintegration of the integrated medical knowledge and experience so as to construct a new system of medical knowledge, which will lead to a new leap in the development of medicine, which is also called data-based medicine or information-based medicine. It covers the following aspects:

- Establishing a platform for preclinical and clinical research in HIM

- Keeping the residents' health records

- Providing information about disease prevention, establishing a system for diagnosis and treatment, and providing analysis of treatment and recovery

- Providing comprehensive knowledge about drug interactions and clinical guidelines

- Integrating specific information of patients

- Establishing strategies of making clinical decisions and communication mechanism for experts in related fields

- Providing methodology for evaluating and predicting the curative effect

- Offering integrated patient health information (electronic health records or electronic medical records of patients from birth to present)

- Developing software assisting independent diagnosis and treatment (such as prevention, diagnosis, risk assessment of health, treatment program, clinical testing and examination, clinical medication and operation, enhancement of patient's confidence)

- Constructing the informationalized environment, including the residents' health records, disease control (through local area network, the Internet of things, and the Internet), family health records, family health information system, home care information system, prehospital emergency care information system, emergency information system, long-term care information system, referral information system, hospital information system (including outpatient station, ward station, admission management system, LIS system, PACS system, the doctor's advice system, ICU monitoring system, and operation management system)

- Keeping electronic medical records, etc.

In this way we could help medical workers discard the dross and select the essential, and get rid of the false and retain the true at the era of knowledge explosion.

\subsubsection{Setting Up HIM Wards}

The integration should be launched among several disciplines as a trial. The disease-oriented integration of internal medicine, surgery, and other related disciplines has already been carried out in some American hospitals over the past few years. Recently, the hospital-within-hospital mode taken in some general hospital in China is a good attempt for HIM. The general wards and intervention wards in some hospitals are to some extent in line with HIM. For example, vascular intervention wards and minimally invasive wards are HIM wards where internal medicinal and surgical techniques are integrated. Strictly speaking, the current general wards are the ones that deal with a variety of diseases whereas ICUs are the wards that deal with acute severe diseases through various techniques. HIM should develop in accordance with ICU mode. The clinic of Preventive Medicine and Health Care Maintenance (CPMHCM), or "Preventive Clinic," should be set up. By integrating this clinic with outpatient clinics, clinical practices such as preventive care and checkup service are organically combined, changing the isolated practice of the vaccination or checkup service. By combining the distinctive advantages of preventive and clinical medicines, such an integrated clinic can provide more comprehensive services for the public, including health education, checkup service, and health records. In this way, we could help patients overcome the difficulty in finding the right department to get their diseases diagnosed and treated due to non-patient-oriented diagnosis and treatment.

\subsubsection{Offering HIM Courses}

The transformation of medical education mode should be accelerated by gradually changing the system-based or discipline-based mode, which requires freshmen to learn with an overall view. The currently advocated training of general practitioners is beneficial to the development of HIM. In the teaching practice of HIM, the integrated courses should be set up for medical students before their internship, accelerating their shift towards doctors equipped with HIM. What's more, the system of cultivating such doctors should also be established. For instance, resident doctors are required to have a prolonged rotation, and newly recruited doctors to receive training in different departments for 3 or 4 years. In this way, they will gradually develop into doctors with the ability of analyzing and solving medical problems. Furthermore, senior practitioners should regularly attend lectures on the development of HIM so as to be equipped with HIM knowledge so that they are able to diagnose and treat patients with HIM knowledge and skills. HIM teaching is by no means the denial of current teaching methods, but the integration and systematization of current teaching methods and contents. In this way, the problem that medical 
students are short of HIM knowledge will be gradually solved, and some misconceptions will also be corrected such as "The top priority for medical students is to become ophthalmologists, next is to become surgeons, and the worst is to become physicians" and "Compared with general practitioners, specialists feel more secure and face less trouble."

\subsubsection{Providing Continuing Education on HIM}

The HIM continuing education can be provided either by the public health organizations at all levels or by some medical colleges and universities headed by academic institutions such as the Chinese Medical Association. First, HIM training should be provided for the medical workers in grade-three class-A hospitals across the country, and then extended gradually to those in grassroots hospitals. Second, HIM knowledge should be added to the Qualification Examination for Licensed Physicians so as to motivate doctors to autonomously learn and apply HIM knowledge. Third, in medical colleges and universities, HIM courses should be offered as compulsory courses in the postgraduate education programs or in on-the-job training for teachers, avoiding the current problem of overspecialization of medicine. In this way, we could solve the problem that a doctor who has been assigned to work in a medical department for lifetime will become a specialized doctor with limited knowledge and skills.

In summary, it takes more than 1000 years for medical science to develop from generalization to specialization. Although specialization has its advantages, it must fit the contexts. Overspecialization fails to help healthcare providers develop a comprehensive view on the truth of life, and the secrets of human body. Without the overall view, a doctor diagnosing a disease is like a blind man figuring out the image of an elephant just by feeling some part of it. Without the overall view, a medical researcher doing medical research is like a man seeing the trees but not the forest. Medical development requires integration, and the fruit of this integration is HIM. HIM, in essence, is to ensure that medical diagnosis and treatment are not organ centered but patient centered and not symptom oriented but disease oriented. HIM requires doctors to act as doctors in the real sense of the term instead of pharmacists, who diagnose and treat patients based on clinical experience rather than laboratory results. HIM is to make sure that medical workers could put the same weight on treatment and nursing, Western medicine and traditional Chinese medicine, and treatment and prevention. However, it should be noted that HIM discipline does not necessarily perform all the medical tasks in a general hospital, nor require HIM practitioners to be competent for all the medical service. The doctors in HIM disci- pline must bear it in mind that they must treat their patients with the guidance of HIM and make them have higher survival rate, longer life span, and better life quality than those treated by other doctors and even themselves in the past. That is the basic requirement for both HIM and healthcare professionals in HIM.

\section{References}

1. Fan D-M. Holistic integrative medicine. Am $\mathrm{J}$ Digest Dis. 2014;1(1):22-36.

2. Fan D. General remarks on holistic integrative medicine (in Chinese). Negative. 2012;3:3-12.

3. Sierpina V, Kreitzer MJ, Anderson R, Hanaway P, Shannon S, Sudak N. The American Board of Integrative and Holistic Medicine: past, present, and future. Explore. 2010;6:192-5.

4. Mantri S. Holistic medicine and the Western medical tradition. Virtual Mentor. 2008;10:177-80.

5. Zuskin E, Lipozencić J, Pucarin-Cvetković J, Mustajbegović J, Schachter N, Mucić-Pucić B, Neralić-Meniga I. Ancient medicine-a review. Acta Dermatovenerol Croat. 2008;16:149-57.

6. Li S, Xu H. Integrative Western and Chinese medicine on coronary heart disease: where is the orientation? Evid Based Complement Alternat Med. 2013;2013:459264.

7. Lam TP, Sun KS. Dilemma of integration with Western medicine - views of traditional Chinese medicine practitioners in a predominant Western medical setting. Complement Ther Med. 2013;21:300-5.

8. Chan E, Tan M, Xin J, Sudarsanam S, Johnson DE. Interactions between traditional Chinese medicines and Western therapeutics. Curr Opin Drug Discov Devel. 2010;13:50-65.

9. Ventegodt S, Andersen NJ, Merrick J. Holistic medicine: scientific challenges. ScientificWorldJournal. 2003;3:1108-16.

10. Mariano C. Holistic nursing as a specialty: holistic nursing-scope and standards of practice. Nurs Clin North Am. 2007;42:165-88.

11. Song JJ, Yan ME, Wu XK, Hou LH. Progress of integrative Chinese and Western medicine in treating polycystic ovarian syndrome caused infertility. Chin J Integr Med. 2006;12:312-6.

12. Liu X, Zhang M, He L, Li Y. Chinese herbs combined with Western medicine for severe acute respiratory syndrome (SARS). Cochrane Database Syst Rev. 2012;10:CD004882.

13. Broadbent A. Philosophy and preventive medicine. Prev Med. 2012;55:575-6.

14. Baetu TM. Genes after the human genome project. Stud Hist Phil Biol Biomed Sci. 2012;43:191-201.

15. Hood L, Rowen L. The human genome project: big science transforms biology and medicine. Genome Med. 2013;5:79.

16. Yip W, Hsiao WC. The Chinese health system at a crossroads. Health Aff. 2008;27:460-8.

17. Fan X, Yang Z, Fan D. Further discussion on holistic integrative medicine (in Chinese). Med Philos. 2013;34:6-11. 27

18. Littman BH, Di Mario L, Plebani M, Marincola FM. What's next in translational medicine? Clin Sci. 2007;112:217-27.

19. Pan SY, Gao SH, Zhou SF, Tang MK, Yu ZL, Ko KM. New perspectives on complementary and alternative medicine: an overview and alternative therapy. Altern Ther Health Med. 2012;18:20-36.

20. Duggal R, Menkes DB. Evidence-based medicine in practice. Int J Clin Pract. 2011;65:639-44.

21. Weisz G. The emergence of medical specialization in the nineteenth century. Bull Hist Med. 2003;77:536-75. 